\title{
La nutracéutica: un enfoque interdisciplinario para la enseñanza de la química orgánica
}

\author{
Recibido: 24-07-2010 | Aceptado: 30-06-2011 \\ The nutraceutical: an interdisciplinary approach \\ to teaching organic chemistry
}

Ana Esperanza Burgos Castellanos*

Rosabel Torrellas Hidalgo**

Resumen: La incorporación de alimentos con propiedades nutracéuticas en la dieta habitual, proporciona una forma sencilla de prevenir enfermedades y mejorar la calidad de vida de la población. La educación se constituye así, en una fuente de conocimiento que puede contribuir a cambiar paradigmas culturales y estimular la utilización de importantes elementos nutricionales autóctonos que muchas veces no son considerados. Resultados experimentales sugieren que existe una relación entre los hábitos alimentarios y la incidencia de muchas enfermedades, entre ellas, el cáncer (Yu, 2010).

La enseñanza de la química orgánica, desde la perspectiva interdisciplinaria de la nutracéutica, se puede constituir en una herramienta para generar competencias científicas, desarrollar el pensamiento complejo e inducir un estilo de vida saludable.

Palabras clave: Nutracéutica, producto natural, enseñanza de la química orgánica, pensamiento complejo, competencias de investigación, interdisciplinariedad, flavonoides, terpenos, polifenoles.
Abstract: Incorporating foods with nutraceutical properties in the diet provides an easy way to prevent diseases and improve the population's quality of life. Education is, therefore, a source of knowledge that can contribute to changing cultural paradigms and encourage the use of indigenous important nutritional elements that are not often considered. Experimental results suggest a relationship between dietary habits and the incidence of many diseases, including cancer. (Yu, 2010). Teaching organic chemistry, from the interdisciplinary perspective of the nutraceutical, may constitute a tool for generating scientific expertise to develop complex thinking and lead a healthy lifestyle.

* Universidad Nacional de Colombia.

* Udana Inc., Houston, Texas, USA. aeburgosc@unal.edu.co, rosabel.torrellas@gmail.com

Keywords: Nutraceutical, natural product, organic chemistry teaching, complex thinking, research skills, interdisciplinary, flavonoids, terpenes, polyphenols. 


\section{Introducción}

Los estándares de competencias y los lineamientos curriculares en ciencias naturales y ciencias sociales del Ministerio de Educación Nacional de Colombia (MEN, 2004), plantean la importancia de profundizar el conocimiento científico para comprender el entorno natural y social. La integración interdisciplinaria de áreas del conocimiento, contribuye a desarrollar la complejidad de la interpretación de los fenómenos (Burgos, 2009).

La enseñanza de la química orgánica constituye una oportunidad para relacionar áreas del conocimiento afines a las ciencias de la vida, desarrollar el pensamiento complejo y generar competencias de investigación. El presente trabajo constituye una reflexión acerca de la contribución de la educación, desde el abordaje interdisciplinario de la nutracéutica, para fomentar una cultura alimentaria que aporte herramientas para mejorar la calidad de vida de la población (MEN, 2004).

\section{Desarrollo}

Actualmente se tienen evidencias de que la alimentación así como el estilo de vida, están directamente relacionados con la prevención o el desarrollo de diversas enfermedades, incluyendo varios tipos de cáncer $(Y u, 2010)$.

La forma de alimentarse en el siglo XXI representa un acto consciente, fundamentado en el conocimiento científico actual, que conjuga el disfrute de una diversidad de sabores con los nutrientes imprescindibles para el organismo y la garantía de la prevención de muchas enfermedades. El tema de la nutrición está experimentando un veloz cambio, siendo el centro del interés, la relación entre la alimentación y las enfermedades crónicas no transmisibles, los efectos sobre las funciones cognitivas e inmunitarias, el desempeño en el trabajo y el rendimiento deportivo. Las personas están cada vez más conscientes de su autocuidado y buscan información relacionada con alimentos que contribuyan a su salud y bienestar (Sivasankaran, 2010; Thibault, 2010).

La nutracéutica constituye una nueva disciplina que estudia las relaciones de los compuestos químicos presentes en los alimentos y la prevención o tratamiento de diversas enfermedades. Su nombre proviene de los términos nutrición y farmacéutica (Kalra, 2003).

Un nutracéutico ha sido definido "como cualquier sustancia que puede ser considerada un alimento o parte de un alimento $y$ que, además de su valor nutricional, aporta beneficios a la salud incluyendo la prevención de enfermedades" (De Felice, 1999).

Cada vez se encuentran pruebas científicas que apoyan la hipótesis de que ciertos alimentos, así como algunos de sus componentes tienen efectos físicos y psicológicos beneficiosos, gracias al aporte de los nutrientes básicos. Las investigaciones en el campo de la nutracéutica actualmente se enfocan en la identificación de los componentes biológicamente activos de los alimentos, que ofrezcan la posibilidad de mejorar las condiciones físicas y mentales, así como de reducir el riesgo a contraer enfermedades (Halliwell, 1996).

Los nutracéuticos actúan de forma beneficiosa sobre una o varias funciones del organismo, pero para obtener esos efectos es preciso integrarlos en la dieta habitual. Además poseen propiedades específicas que pueden aprovecharse en las distintas fases de la vida y se considera que algunos 
La nutracéutica: un enfoque interdisciplinario para la enseñanza de la química orgánica Ana Esperanza Burgos Castellanos | Rosabel Torrellas Hidalgo

de ellos podrían contribuir, por ejemplo, a reducir el riesgo de osteoporosis, afecciones cardíacas, prevenir algunos tipos de cáncer, entre otros beneficios. Algunos compuestos químicos encontrados en los alimentos, útiles para la prevención y el tratamiento de enfermedades, incluyen flavonoides, polifenoles, terpenos, vitaminas, oligoelementos y lípidos, entre otros (Pandey, 2010).

Desde el punto de vista didáctico, la búsqueda de elementos que permitan la vinculación del conocimiento con competencias cognitivas, ética, emociones, aptitudes y actitudes para la toma de decisiones individuales y profesionales en el contexto de la vida diaria, constituye hoy una necesidad en el campo de la práctica docente para el encuentro de la ciencia y la cultura (Rychen y Tiana, 2004).

La nutracéutica reúne un escenario de varias áreas del conocimiento que incluyen la química orgánica, biología, bioquímica, medicina, nutrición, farmacología y farmacia, entre otras. Esta particularidad proporciona una interesante aproximación para la enseñanza de la química del grado 11 de educación secundaria y de los primeros semestres universitarios en Colombia, al correlacionar áreas científicas con el cuidado del cuerpo mediante una alimentación saludable.

Se propone un abordaje interdisciplinario para el estudio de la química orgánica, que despierte la motivación en los estudiantes por los descubrimientos recientes en el campo de los alimentos con propiedades para prevenir enfermedades. Así, el estudiante podrá participar activamente en su proceso de aprendizaje, al estudiar estructuras químicas de tres grupos de compuestos orgánicos con propiedades farmacológicas.

Los grupos de compuestos químicos propuestos, son los flavonoides, polifenoles y terpenos. Su escogencia se fundamenta en el hecho de que ellos están presentes en una variedad de alimentos de amplio consumo en la población y sus características químicas, pueden ser explicadas a través de varios temas del plan de estudio del último año de la enseñanza secundaria y de los primeros semestres universitarios. Así, la integración de los conocimientos podrá facilitar, dentro del contexto de la exploración de las ideas, la motivación en los estudiantes para la investigación y la correlación de aspectos químicos con la biología, la bioquímica, la bioinorgánica, la química medicinal, la educación ambiental, educación para la salud y la educación para el consumidor.

Se proponen actividades de enseñanza y aprendizaje variadas, que conjuguen la parte interdisciplinar con estrategias didácticas que incluyan actividades individuales, socializaciones en pequeños grupos y concertaciones que serán complementadas por el docente. Además se plantean actividades de reflexión, de lectura y de búsqueda e interpretación de información relevante de artículos o revistas científicas que relacionen la importancia de la alimentación y la salud. Además se investigará, las principales enfermedades asociadas a la alimentación, las estructuras químicas de algunos flavonoides, polifenoles y terpenos, los alimentos que contienen estos compuestos químicos y su utilidad terapéutica. Posteriormente, cada estudiante realizará con su grupo y con el profesor, la discusión de las informaciones obtenidas, los beneficios de los nutracéuticos, su difusión en el mercado de alimentos y la importancia de una alimentación sana, dentro de un estilo de vida saludable, como forma de cuidarse a si mismo y prevenir enfermedades. Estas actividades enriquecerán el conocimiento y permitirán al docente ahondar en el concepto de grupo funcional, discutir las estructuras de los compuestos aromáticos, las propiedades físicas y químicas y los tipos de enlaces derivados de la 
hibridización del carbono, entre otros aspectos. Así, el estudiante podrá desarrollar la curiosidad, la persistencia, la apertura mental, la capacidad de pensamiento crítico, la cultura alimentaria y la disposición para el trabajo en equipo, entre otras habilidades. La interacción compleja entre las competencias de investigación, los conceptos de química orgánica y la nutracéutica, contribuirán a desarrollar en los estudiantes una construcción mental capaz de interpretar y relacionar conceptos cientíicos en la cotidianidad.

La estrategia planteada para la enseñanza de la química orgánica, utilizando el enfoque interdisciplinario que proporciona la discusión de los tres grupos de compuestos señalados anteriormente, contribuiría a profundizar varios temas del programa de la materia. Así, tomaría sentido práctico la explicación de la configuración electrónica del carbono y su hibridación $\mathrm{sp}, \mathrm{sp}^{2}$ y $\mathrm{sp}^{3}$, las estructuras moleculares derivadas de los enlaces simples, doble y triples, la geometría molecular, los tipos de isomería y la identificación de los grupos funcionales más importantes, para permitir la discusión de las estructuras químicas de los compuestos y la predicción de propiedades físicas y químicas resaltantes, tales como solubilidad, puntos de fusión y reactividad, entre otros.

De otra parte, la discusión del concepto de isomería, en función de los diferentes arreglos espaciales, puede constituirse en un abordaje para la compresión de la importancia de la quiralidad en la naturaleza. Así, se abriría un espacio para un acercamiento conceptual a la teoría de los receptores, al mostrar que unos isómeros ópticos presentan efectos biológicos y otros no. Finalmente, la integración interdisciplinaria alcanzada a través de la experiencia didáctica, podría propiciar en los estudiantes, el interés por continuar profundizando el conocimiento de la química orgánica.
A continuación se presentan algunos representantes del grupo de los flavonoides (Figuras 1-3), polifenoles (Figura 4-6) y terpenos (Figura 7), con el fin de implementar la alfabetización científica en la química orgánica y familiarizar al estudiante con estructuras química, desde las perspectivas anteriormente señaladas.

\section{Flavonoides}

Los flavonoides son la clase de polifenoles más abundante y extendida del mundo vegetal. Se encuentran en distintas partes de las plantas vasculares. En 1937 el científico húngaro Albert Szent-Györgyi, ganador del premio Nobel de Fisiología y Medicina, determinó sus propiedades protectoras vasculares y les dio el nombre de vitamina $P$ (Szent-Györgyi, 1965).

Hasta ahora se han encontrado en estos productos naturales, propiedades antioxidantes, antiinflamatorias, antiagregantes, antihemorrágicas, vasodilatadoras, antineoplásicas, antivirales, antibacterianas, antialérgicas y hepatoprotectoras (Tripoli, 2007).

Actualmente, sus propiedades son objeto de importantes investigaciones biomédicas. En función de estas actividades, los flavonoides son considerados hoy como potenciales agentes terapéuticos frente a una amplia variedad de enfermedades. Desde el punto de vista químico, los flavonoides constituyen un gran número de productos naturales de bajo peso molecular, la mayoría con estructura de fenil-benzo- $\gamma$ pirano (o fenil- $\gamma$-cromona), productos del metabolismo secundario vegetal (Figura 1).

En las plantas, los flavonoides se encuentran en estado libre o más frecuentemente en forma de glicósidos flavonólicos. Los glicósidos son generalmente solubles en agua, 
mientras que sus agliconas, la parte no asociada al glicósido, lo son sólo ligeramente. Existe una relación entre la actividad biológica de los flavonoides y su estructura química, en particular con el patrón de oxhidrilación en los anillos de la estructura (Heima, 2002).

La investigación biomédica actualmente describe, interesantes actividades biológi- cas de los flavonoides, relacionadas con la patología cardiovascular (Russo, 2006; De Luis, 2008).

Estudios in vitro e in vivo han demostrado la acción relajante de los flavonoides sobre el músculo liso vascular y en la regulación del flujo sanguíneo y del tono vascular. (Ajay, 2003).<smiles></smiles>

Fenil-benzo- $\gamma$-Pirano<smiles>O=C(/C=C/c1ccccc1)c1ccccc1</smiles>

Chalcona<smiles>O=C1CC(c2ccccc2)Oc2ccccc21</smiles>

Flavonona<smiles>O=c1c(-c2ccccc2)coc2ccccc12</smiles>

Isoflavona<smiles>O=c1c(O)c(-c2ccccc2)oc2ccccc12</smiles>

Flavonol<smiles>O=C1c2ccccc2OC(c2ccccc2)C1O</smiles>

Flavononol<smiles>O=c1cc(-c2ccccc2)oc2ccccc12</smiles>

Flavona<smiles>OC1Cc2ccccc2OC1c1ccccc1</smiles>

Flavanol<smiles>Oc1cc2ccccc2[o+]c1-c1ccccc1</smiles>

Antocianidina

Figura 1. Estructuras básicas de los flavonoides. 
Los flavonoides miricetina, kaempferol, luteolina y apigenina aislados en Apium graveolens rapaceum (apio o célery) han mostrado actividades antioxidantes vasoconstrictora, anticancerígena, antiinflamatorias, antibacterianas, inmuno-estimulantes, antivirales y diurética. Diosmina, una flavonona que fortalece la pared vascular y rutina, un glicósido flanonol que se encuentran en el té verde, cebolla, vino tinto y varios vegetales, se usan terapéuticamente para disminuir la fragilidad capilar y la insuficiencia venosa.<smiles>O=c1c(O)c(-c2cc(O)c(O)c(O)c2)oc2cc(O)cc(O)c12</smiles>

Miricetina<smiles>O=c1c(O)c(-c2ccc(O)cc2)oc2cc(O)cc(O)c12</smiles>

Kaempferol<smiles>COc1ccc(-c2cc(=O)c3c(OC)c(OC)c(OC)c(OC)c3o2)cc1</smiles>

Iangeretına
Quercetina, un poderoso antioxidante con propiedad anticancerígena, antiinflamatoria, antiartrítica y protectora de la función cardiovascular, se encuentra en los cítricos y muchas frutas y vegetales. Hesperidina, rutina, tangeretina, diosmetina, narirutina, neohesperidina que se encuentran en la cáscara de los cítricos como naranja y el limón, son flavononas con efectos antioxidantes y protectores cardiovasculares que mejoran los perfiles lipídicos en sangre, ver la figura 2; (De Pascual-Teresa, 2010; Androutsopoulos, 2010).

\section{Quercetina}<smiles>O=c1cc(-c2ccc(O)cc2)oc2cc(O)cc(O)c12</smiles>

Apigenina<smiles>O=c1cc(-c2ccc(O)c(O)c2)oc2cc(O)cc(O)c12</smiles>

Luteolina 
<smiles>COc1ccc(-c2cc(=O)c3c(O)cc(O[C@@H]4O[C@H](CO[C@@H]5O[C@@H](C)[C@@H](O)[C@H](O)[C@H]5O)[C@@H](O)[C@H](O)[C@H]4O)cc3o2)cc1O</smiles>

Diosmina<smiles>C[C@H]1O[C@H](OC[C@H]2O[C@H](Oc3c(-c4ccc(O)c(O)c4)oc4cc(O)cc(O)c4c3=O)[C@H](O)[C@H](O)[C@@H]2O)[C@H](O)[C@@H](O)[C@@H]1O</smiles>

Rutina<smiles>COc1ccc(-c2cc(=O)c3c(O)cc(O)cc3o2)cc1O</smiles>

Diosmetina<smiles>COc1ccc(C2CC(=O)c3c(O)cc(O[C@@H]4O[C@H](CO)[C@@H](O)C(O)[C@H]4O[C@H]4O[C@H](C)[C@@H](O)[C@H](O)[C@H]4O)cc3O2)cc1O</smiles>

Neohesperidina<smiles>C[C@H]1O[C@H](OC[C@H]2O[C@H](Oc3cc(O)c4c(c3)OC(c3ccc(O)cc3)CC4=O)[C@H](O)[C@H](O)[C@@H]2O)[C@H](O)[C@H](O)[C@@H]1O</smiles>

Narirutina

Figura 2. Algunos flavonoides que presentan actividades biológicas. 
Un importante grupo de isoflavonoides que actualmente están siendo estudiados, lo constituyen los fitoestrógenos derivados de la soja. Se sospecha que ejercen efectos benéficos para la salud, en particular para la mujer (Riesco, 2010; Bolca, 2010).

Los fitoestrógenos se hallan en muchos vegetales, que son consumidos habitualmente en la dieta humana. Soja, alfalfa, semillas de linaza, son las fuentes alimenticias más significativas de isoflavonas. Los más conocidos como nutracéuticos son la genesteína, de acción antineoplásica, inhibidor enzimático, anticancerígeno y estrogénico y la diadzeína también de acción estrogénica (Figura 3). Estudios epidemiológicos indican que el con-<smiles>O=c1c(-c2ccc(O)cc2)coc2cc(O)cc(O)c12</smiles>

Genesteína sumo de dietas ricas en cereales, legumbres y soja, disminuye el riesgo de determinados cánceres, como el de mama, colon y próstata, así como de enfermedades coronarias. Estudios han demostrado que las poblaciones de mujeres asiáticas, en particular japonesas, quienes son importantes consumidoras de productos de soja, presentan una menor incidencia y prevalencia de las enfermedades señaladas antes, que los grupos estudiados de mujeres europeas y norteamericanas (Ward, 2010; Riesco, 2010). También se ha indicado que los fitoestrógenos son biomarcadores de una dieta saludable, encontrándose una relación inversa entre los niveles de fitoestrógenos en la orina y el riesgo de cáncer de mama (Hsu, 2010).

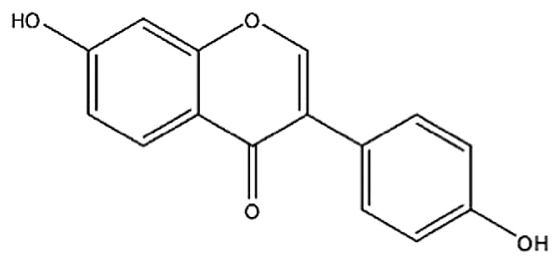

Daidzeína

Figura 3. Estructura de algunos fitoestrógenos de importante actividad farmacológica.

\section{Polifenoles}

Algunos polifenoles encontrados en el vino tienen efecto vasodilatador dependiente del endotelio. Resveratrol (Figura 4) es un poderoso antioxidante que se encuentra en el vino, mosto, nueces, arándanos, entre otros. Químicamente es una fitoalexina (sustancia que produce la planta ante distintas situaciones de estrés). Además se le asocia propiedades como antiinflamatorio, antineoplásico, antioxidante, inhibidor enzimático, inhibidor de la agregación plaquetaria, antimutagénico y antienvejicimiento (Frémont, 2000). De otra parte, los estudios in vivo, con ratones obesos han mostrado que el tratamiento con resveratrol, aumenta el período de supervivencia cuando son alimentados con una dieta alta en calorías (Baur, 2006). 


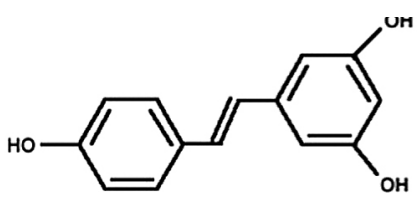

Figura 4. Estructura química del resveratrol.

Otro grupo de polifenoles no flavonoides que actualmente están siendo intensamente estudiados, son los curcuminoides encontrados en la raíz de la Curcuma longa L. En la India, y en otros países asiáticos, por más de 2000 años, esta planta ha sido utilizada como condimento y para tratar diversas dolencias. Se ha encontrado que es útil para tratar la enfermedad de Alzheimer, combatir desórdenes digestivos, para la prevención del cáncer, prevención de cataratas, uveítis anterior crónica, colesterol alto, protección al hígado, dolor menstrual, esclerosis múltiple, osteoartritis y artritis reumatoide (Figura 5). Al presente se desarrollan importantes investigaciones para determinar la actividad anticancerígena y anti-alzheimer de los curcuminoides (Ishrat, 2009; Cashman, 2008). Estos compuestos constituyen el pigmento amarillo obtenido de la raíz de Curcuma longa L. tienen un espectro farmacológico que incluye actividad antioxidante, anti-inflamatoria, inhibición enzimática, antiviral, hipocolesteromiante y acción anticancerígena (Joe, 2004).

De gran interés nutracéutico actual, son los polifenoles Epicatequina y Teaflavina, (Figura 6) que se encuentran en el té verde de las hojas de la planta Camellia sinensis. A ellos se les atribuyen importantes actividades farmacológicas, entre las que se destacan sus efectos anticancerígenos y antioxidantes (Loke, 2010; Lorenz, 2009).<smiles>COc1cc(/C=C/C(=O)CC(=O)/C=C/c2ccc(O)c(OC)c2)ccc1O</smiles>

Curcumina<smiles>COc1cc(/C=C/C(=O)CC(=O)/C=C/c2ccc(O)cc2)ccc1O</smiles>

Demetoxicurcumina<smiles>O=C(/C=C/c1ccc(O)cc1)CC(=O)/C=C/c1ccc(O)cc1</smiles>

Bisdemetoxicurcumina

Figura 5. Curcuminoides encontrados en la raíz de Curcuma longa $\mathrm{L}$.<smiles>Oc1cc(O)c2c(c1)O[C@H](c1ccc(O)c(O)c1)[C@H](O)C2</smiles>

Epicatequina<smiles>O=C(C[C@@H]1Oc2cc(O)cc(O)c2C[C@H]1c1cc(O)c(O)c2c(=O)c(O)cc([C@@H]3Oc4cc(O)cc(O)c4CC3OC(=O)c3cc(O)c(O)c(O)c3)cc12)c1cc(O)c(O)c(O)c1</smiles>

Teaflavina

Figura 6. Polifenoles del té verde. 


\section{Terpenos}

Los terpenos son un grupo importante de productos naturales, que aunque tienen estructuras químicas muy distintas, se originan en la planta a partir de la condensación de un número variable de unidades de isopreno (Figura 7).

Los carotenoides constituyen un grupo de terpenos de interés nutracéutico. Su incorporación en la dieta habitual conduce a prevenir enfermedades degenerativas. Ejemplos de carotenoides, que actualmente están siendo objeto de estudios por sus propiedades farmacológicas son la Capsantina, y la Capsorrubina, (Figura 7), obtenidas del extracto del fruto de pimentón Capsicum annuum L. (Krinski, 2005).
El licopeno, el pigmento rojo del tomate que presenta comprobada actividad antioxidante, está siendo estudiado por sus propiedades para prevenir las enfermedades cardivasculares y neurodegenerativas, la hipertensión y para el tratamiento del cáncer de próstata (Waliszewski, 2010). La bixina y la norbixina que se obtienen de la extracción de la cubierta exterior de las semillas de Bixa orellana L., conocida popularmente como bija, onoto, achiote, urucú, les ha sido encontrada actividad antimutagénica, antioxidante y antibacterial (Junior, 2005).

El eugenol (Figura 7) presente en la especie clavo de olor, con potente actividad antibacterial, constituye otro de los tantos nutracéuticos terpénicos que aportan importantes beneficios a la salud (Devi, 2010).

Figura 7. Algunas estructuras de compuestos terpenoides.<smiles>C=CC(=C)C</smiles>

Isopreno Isopentadieno, 2-Metilbutadieno<smiles>CC1=C(/C=C(C)/C=C/C=C/C(C)=C/C=C/C=C(C)/C=C/C=C(C)/C=C/C=C(\C)C2=C(C)CCCC2(C)C)C(C)(C)CCC1</smiles>

$\beta$-caroteno

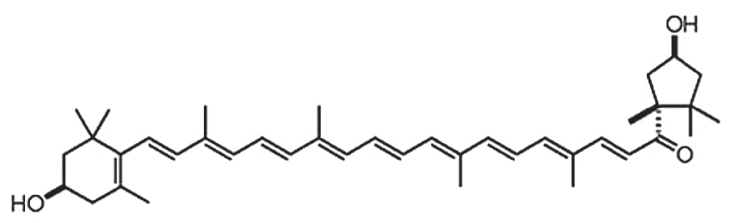

Capsantina<smiles>C=CCc1ccc(O)c(OC)c1</smiles>

Eugenol 

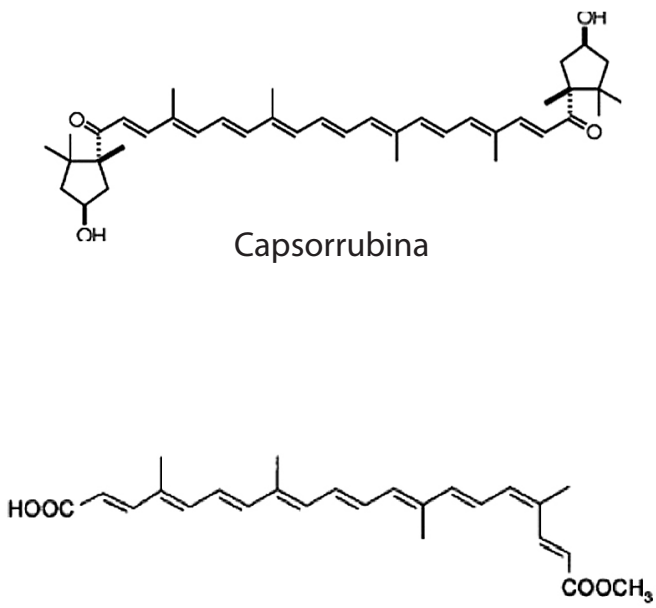

Bixina

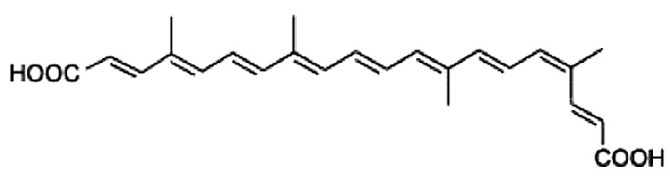

Norbixina

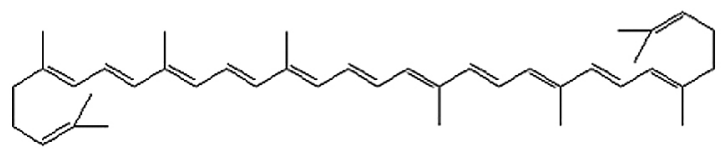

Licopeno

Figura 7. Algunas estructuras de compuestos terpenoides.

\section{Conclusiones}

Los estudios actuales de la nutracéutica constituyen un valioso aporte para la prevención y tratamiento de diversas enfermedades que se traducen en una mejor calidad de vida. La enseñanza de la química orgánica a través del abordaje interdisciplinario de la nutracéutica, contribuye a profundizar el pensamiento complejo, generar competencias científicas y reflexionar acerca de la importancia de una alimentación consciente, al valorar la utilización de los recursos naturales autóctonos. 


\section{Bibliografía}

Ajay, M.; Gilani, A. U.; Mustafa, M. R. (2003). Effects of flavonoids on vascular smooth muscle of the isolated rat thoracic aorta. Life Sci, 74, pp. 603-612.

Androutsopoulos, V. P.;Papakyriakou, A.; Vourloumis, D.; Tsatsakis, A. M.; Spandidos, D. A. (2010). Dietary flavonoids in cancer therapy and prevention: substrates and inhibitors of cytochrome P450 CYP1 enzymes. Pharmacol Ther. Apr;126(1):9-20.

Baur, J. A. (2006), Resveratrol improves health and survival of mice on a high-calorie diet, Nature, 444, pp. 337-342.

Baur, J. A.; Sinclair, D. A. (2006). Therapeutic potential of resveratrol: the in vivo evidence, Nature Reviews Drug Discovery, 5, pp. 493-506.

Bolca, S.; Urpi-Sarda, M.; Blondeel, P.; Roche, N.; Vanhaecke, L.; Possemiers, S.; Al-Maharik, N.; Botting, N.; De Keukeleire, D.; Bracke, M.; Heyerick, A.; Manach, C.; Depypere, H. (2010). Disposition of soy isoflavones in normal human breast tissue, Am J Clin Nutr, 91, 4, pp. 976-84.

Burgos, A. E; Torrellas, R. (2009). Educación Superior y Pensamiento Complejo en la Sociedad del Conocimiento. Tecné, Episteme y Didaxis: TED No. Extraordinario.

Cashman, J. R.; Ghirmai, S.; Abel, K.J.; Fiala, M. (2008). Immune defects in Alzheimer's disease: new medications development, $B M C$ Neurosci, 3, 9Suppl2, S13.

De Felice, S. L. (1999). The nutraceutical revolution: its impact on food industry R\&D. Trends Food Sci \& Technol, 6, 2, 59-6.

De Luis, D. A.; Aller, R. (2008). Papel de los flavonoides del té en la protección cardiovascular, An Med Interna, 25, pp. 105-107.

De Pascual-Teresa, S.; Moreno, D. A.; GarcíaViguera, C. (2010). Flavanols and anthoc- yanins in cardiovascular health: a review of current evidence, Int J Mol Sci, 11, 4, 1679-703.

Devi, K. P.; Nisha, S. A, Sakthivel, R.; Pandian, S. K. (2010). Eugenol (an essential oil of clove) acts as an antibacterial agent against Salmonella typhi by disrupting the celIular membrane. J Ethnopharmacol. Jul 6;130(1):107-15.

Frémont, L. (2000). Biological effects of resveratrol, Life Sciences, 66, 8, pp. 663-673.

Halliwell, B. (1996). Antioxidants in human health and disease, Ann Rev Nutr, 16, pp. 33-50.

Heima, K. E.; Tagliaferroa, A.; Bobilya, D. J. (2002). Flavonoid antioxidants: chemistry, metabolism and structure-activity relationships. J Nutr Biochem 10, pp. 572-584.

Hsu, A.; Bray, T. M.; Helferich, W. G.; Doerge, D. R.; Ho, E. (2010). Differential effects of whole soy extract and soy isoflavones on apoptosis in prostate cancer cells, Exp Biol Med (Maywood), 235, 1, 90-7.

Ishrat, T.;Hoda, M. N.; Khan, M. B.; Yousuf, S.; Ahmad, M.; Khan, M. M.; Ahmad, A.; Islam, F. (2009). Amelioration of cognitive deficits and neurodegeneration by curcumin in rat model of sporadic dementia of Alzheimer's type (SDAT). Eur Neuropsychopharmacol, $19,9,636-47$.

Joe, B.; Vijaykumar, M.; Lokesh, B. R. (2004). Biological properties of curcumin-cellular and molecular mechanisms of action, Crit Rev Food Sci Nutr, 44, 2, pp. 97-111.

Júnior, A. C.; Asad, L. M; Oliveira, E. B.; Kovary, K.; Asad, N. R.; Felzenszwalb, I. (2005). Antigenotoxic and antimutagenic potential of an annatto pigment (norbixin) against oxidative stress. Genet Mol Res. Mar 31;4(1):94-9.

Kalra, E.K. (2003). Nutraceutical-definition and introduction. AAPS PharmSci.; 5(3):E25. 
La nutracéutica: un enfoque interdisciplinario para la enseñanza de la química orgánica Ana Esperanza Burgos Castellanos | Rosabel Torrellas Hidalgo

Krinsky, N. I.; Johnson, E. J. (2005). Carotenoid actions and their relation to health and disease. Mol Aspects Med. Dec; 26(6): 459-516.

Loke, W. M.; Proudfoot, J. M.; Hodgson, J. M.; McKinley, A. J.; Hime, N.; Magat, M.; Stocker, R.; Croft, K. D. (2010). Specific dietary polyphenols attenuate atherosclerosis in apolipoprotein E-knockout mice by alleviating inflammation and endothelial dysfunction. ArteriosclerThromb Vasc Biol, 30, 4, pp. 749-57.

Lorenz, M.; Urban, J.; Engelhardt, U.; Baumann, G.; Stangl, K.; Stangl, V.( 2009). Green and black tea are equally potent stimuli of NO production and vasodilation: new insights into tea ingredients involved. Basic Res Cardiol. Jan;104(1):100-10

Ministerio de Educación Nacional (2004). Estándares básicos de competencias en ciencias naturales y ciencias sociales.

Ministerio de Educación Nacional. (2004). Serie lineamientos curriculares.

Pandey, M.; Verma, R .K.; Saraf, S.A. (2010). Nutraceuticals: new era of medicine and health. Asian Journal of Pharmaceutical and Clinical Research, 3, 1. 11-15.

Riesco, E.; Aubertertin-Leheudre, M.; Maltais, M. L.; Audet, M.; Dionne, I. J. (2010). Synergic effect of phytoestrogens and exercise training on cardiovascular risk profile in exercise-responder postmenopausal women: a pilot study, Menopause, SepOct;17(5):1035-9.

Russo, R. O.; Sanchez, S. (2006). Los flavonoides en la terapia cardiovascular, Rev. Costarric. Cardiol, 8, 1, pp. 13-18.
Rychen, D. S.; Tiana, A. (2004). Developing Key Competencies in Education: Some Lessons from International and National Experience, Geneva: UNESCO-IBE, Studies in Comparative Education.

Sivasankaran, S. (2010). The cardio-protective diet. Indian J Med Res. Nov; 132(5):608-16.

Szent-Györgyi, A. (1965). Oxidation, Energy Transfer, and Vitamins, Nobel Lectures, Physiology or Medicine, Amsterdam: Elsevier Publishing Company. 1922-1941.

Thibault, R.; Dupertuis, Y. M.; Belabed, L.; Pichard, C. (2010). Nutrition and physical activity: two targets for cancer prevention, Rev Med Suisse, 6, 250, pp. 1046-1048.

Tripoli, E.; Guardia, M.; Giammanco, S.; Majo, M. (2007). Citrus flavonoids: Molecular structure, biological activity and nutritional properties: A review, Food Chemistry, 104, 2, pp. 466-479.

Yu, X.; Zhang T.; Zhang, H.;Hu, A.; Hu, Y.; Guo, W.; Wang, Y. (2010). Comparison of lifestyle and living environment among high risk immigrant and low risk host residents: implications for esophageal cancer etiology. Asian Pac J Cancer Prev., 11(6):1827-31.

Waliszewski, K.N.; Blasco, G. (2010). Propiedades nutraceúticas del licopeno, salud pública de Mex, 52, 3, pp. 254-65.

Ward, H.A.; Huhnle, G.G. (2010). Phytoestrogen consumption and association with breast, prostate and colorectal cancer in EPIC Norfolk, Arch Biochem Biophys. Sep 1;501(1):170-5. 